\title{
COMMUTATIVE RINGS WHOSE PRIME IDEALS ARE RADICALLY PERFECT
}

\author{
V. ERDOĞDU AND S. HARMAN
}

\begin{abstract}
The main objective of this paper is to relate the height and the number of generators of ideals in rings that are not necessarily Noetherian. As in $[\mathbf{1 0}, \mathbf{1 1}]$, we call an ideal $I$ of a ring $R$ radically perfect if among the ideals of $R$ whose radical is equal to the radical of $I$ the one with the least number of generators has this number of generators equal to the height of $I$. This is a generalization of the notion of set theoretic complete intersection of ideals in Noetherian rings to rings that need not be Noetherian. In this work, we determine conditions on a ring $R$ so that the prime ideals of $R$ and also those of the polynomial rings $R[X]$ over $R$ are radically perfect. In many cases, it is shown that the condition of prime ideals of $R$ or that of $R[X]$ being radically perfect is equivalent to a form of the class group of $R$ being torsion.
\end{abstract}

1. Introduction. One of the interesting research topics in commutative algebra is to relate the number of generators of an ideal to its height. The link between the number of generators to the height of an ideal is extensively investigated in the literature in the cases when the underlying ring is Noetherian (see, e.g., $[\mathbf{4}, \mathbf{1 7}, 18$ and the references therein]). The most noted question in this regard asks whether each height two ideal of the polynomial ring $K[X, Y, Z]$ is radically perfect, where $K$ is a field of characteristic zero. Since $K[X, Y, Z]$ is both a Hilbert domain and a Krull domain, which at the same time can be regarded as a polynomial ring in one variable over such rings, we are naturally led to consider the notion of radically perfectness of ideals in polynomial rings mostly over Hilbert and Krull domains. That is, with the help of our new definition of radically perfectness of an ideal, we were able to relate the height and the number of generators (up to radical) of an ideal in rings that are not necessarily Noetherian.

\footnotetext{
2010 AMS Mathematics subject classification. Primary 13B25, 13B30, 13C15, 13C20, 13F05, 13F20, Secondary 13A15, 13A18, 14H50.

Keywords and phrases. Radically perfectness, coprime packedness, polynomial rings, Hilbert domains, Krull domains, Prüfer domains.

The second author is the corresponding author.

Received by the editors on June 29, 2011, and in revised form on April 10, 2012.

DOI:10.1216/JCA-2013-5-4-527 Copyright (C)2013 Rocky Mountain Mathematics Consortium
} 
The layout of this work is as follows. In Section 2, we recall some statements from [11] and also generalize Theorem 2.2 of [11]. In Section 3, we examine radically perfectness of ideals in Hilbert domains of finite character and, among other things, we prove that over a Noetherian Hilbert domain $R$ of finite character that contains a field of characteristic zero, each prime ideal of $R[X]$ is radically perfect if and only if $R$ is a Dedekind domain with torsion ideal class group. We also show that, in a Hilbert domain $R$ in which each nonzero ideal is contained in finitely many maximal ideals, then each invertible maximal ideal is radically perfect if and only if $\operatorname{CopRad}(R)$ is torsion, where CopRad $(R)$ denote the multiplicative semigroup generated by the set of pairwise coprime radical ideals of $R$ that are invertible. In Section 4, we examine radically perfectness of prime ideals in Krull domains, and our main result in this section states that in a two-dimensional Krull domain $R$ of finite character, each prime ideal of $R$ is radically perfect if and only if the divisor class group of $R$ is torsion. We also examine radically perfectness of maximal ideals in Int $(R)$, the ring of integer valued polynomials over $R$, and show that radically perfectness of maximal ideals of $\operatorname{Int}(R)$ implies that each prime ideal $P$ of $R$ is the radical of an ideal generated by at most ht $(P)+1$ elements. In Section 5, we show that, if each prime ideal of $R[X]$ over a finitedimensional Prüfer domain $R$ of finite character is radically perfect, then $R$ is of dimension at most two and in some special cases is of dimension one. Finally, in Section 6, we relate the notions of radically perfectness and coprime packedness of prime ideals of various rings. In particular, we show that in a finite-dimensional Hilbert domain $R$, if MaxSpec $R$ is coprimely packed, then each maximal ideal $M^{*}$ of $R[X]$ is the radical of an ideal generated by two elements. We also show that, over a Prüfer domain $R$ with coprimely packed set of maximal ideals which has the property that each maximal ideal of it is finitely generated, then for each maximal ideal $M$ of $R$ the prime ideal $M R[X]$ of $R[X]$ is radically perfect if and only if $R$ is a Dedekind domain with torsion ideal class group.

Throughout, $R$ will always denote a commutative ring with identity, and $R[X]$ will denote the polynomial ring over $R$. Also, by the dimension of $R$, we will mean the Krull dimension of $R$.

2. Finite generation of ideals in rings of finite character up to Jacobson radical. We begin this section by recalling that 
a $\operatorname{ring} R$ is of finite character if each nonzero element is contained in only finitely many maximal ideals. (This is the usual definition of finite character used in the literature which differs from the one used in [11]). Before stating our main result of this section, we borrow some of the statements from $[\mathbf{1 1}]$ that are used in the sequel.

Definition 2.1 (see [11]). Let $R$ be a ring and $I$ an ideal of $R$. We denote by $j-\operatorname{rad}(I)$ the intersection of all maximal ideals of $R$ containing $I$, and by $j_{\max }-\operatorname{rad}(I)$, the intersection of all maximal ideals of $R$ of maximal height that contains $I$.

Proposition 2.2 [11, Proposition 2.1]. Let $R$ be a ring in which each nonzero element is contained in only finitely many maximal ideals. Then each maximal ideal of $R$ is the $j$-radical of an ideal generated by two elements.

Proof. Let $M$ be any maximal ideal of $R$ and $u$ a non-zero element of $M$. Then $u$ is contained in finitely many maximal ideals, and let $M=M_{1}, M_{2}, \ldots, M_{n}$ be the only maximal ideals of $R$ containing $u$. It is clear that there is an element $v$ in $M=M_{1}$ and an element $a \in$ $\bigcap_{k=2}^{n} M_{k}$ such that $v+a=1$ in $R$ and that $M=M_{1}=j-\operatorname{rad}(u, v)$.

The following is a generalization of Theorem 2.2 of [11].

Theorem 2.3. Let $R$ be a finite-dimensional integral domain of finite character and $R[X]$ the polynomial ring over $R$. Then each maximal ideal $M^{*}$ of $R[X]$ of maximal height is the $j$-radical of an ideal generated by three elements and $j_{\max }$-radical of an ideal generated by two elements.

Proof. Let $M^{*}$ be a maximal ideal of $R[X]$ of maximal height such that $M^{*} \cap R=M$. We first show that $M$ is maximal in $R$. To do so, we note that there is a chain of prime ideals of $R[X]$ contained in $M^{*}$ passing through $M R[X]$ of length equal to the dimension of $R[X]$. Since there cannot be a chain of three distinct prime ideals of $R[X]$ having the same contraction in $R$, it follows that there cannot be a prime ideal between $M R[X]$ and $M^{*}$ in $R[X]$. If $M$ is not maximal, then $M$ 
would be contained in a maximal ideal $N$ of $R$ and that would give a longer chain of prime ideals in $R[X]$ of length equal to $\operatorname{dim} R[X]+1$. This contradiction shows that $M$ is maximal in $R$ and, therefore, by [16, Theorem 28], $M^{*}=(M, f)$ for some monic polynomial $f$ in $M^{*}$. Now use the proof of Proposition 2.2 to see that $M^{*}$ contains $j-\operatorname{rad}(u, v, f)$, where $M=j-\operatorname{rad}(u, v)$ for some elements $u$ and $v$ in $M$. Let $Q^{*}$ be any maximal ideal of $R[X]$ containing the ideal $(u, v, f)$. Then, since $Q^{*}$ is maximal and contains a monic polynomial, it follows that $Q^{*} \cap R$ is a maximal ideal of $R$ containing $u$ and $v$ and so has to be the maximal ideal $M$. Therefore, $M^{*}=Q^{*}$ in $R[X]$ and so $M^{*}=j-\operatorname{rad}(u, v, f)$. Next, by adapting the proof of Theorem 2.2 of [11], we obtain $M^{*}=j_{\max }-\operatorname{rad}(u, g)$ for some polynomial $g$ in $M^{*}$ where $g=v+a f$ and $u, v$ and $a$ are as in the proof of Proposition 2.2.

\section{Radically perfect prime ideals in Hilbert domains of}

finite character. In this section, we show that the condition of a finite character on a Hilbert domain $R$ implies that each maximal ideal $M^{*}$ of $R[X]$ is the radical of an ideal generated by two elements and that radically perfectness of maximal ideals of $R$ is equivalent to the condition that CopRad $(R)$ is torsion.

Definition 3.1. An ideal $I$ of a ring $R$ is radically perfect if the height of $I$ is equal to the infimum of the number of generators of ideals of $R$ whose radical is equal to the radical of $I$.

Proposition 3.2. A Hilbert domain $R$ of finite character is of dimension one and each maximal ideal of it is the radical of an ideal generated by two elements.

Proof. Let $P$ be a nonzero prime ideal of $R$ and $a$ a nonzero element of $P$. Then, since $R$ is a Hilbert domain of finite character, $\operatorname{rad}(a)=$ $M_{1} \cap \cdots \cap M_{n}$ for some maximal ideals $M_{1}, \ldots, M_{n}(1 \leq i \leq n)$. But then it follows that $P$ contains at least one of the maximal ideals $M_{i}$ for some $i$, and so $P$ is maximal. Therefore, $R$ is of dimension one. Now if $M$ is a maximal ideal of $R$, then by Proposition 2.2, there are elements $u$ and $v$ in $M$ such that $M=j-\operatorname{rad}(u, v)$. Since $R$ is a Hilbert domain, for any ideal $I$ of $R, j-\operatorname{rad}(I)=\operatorname{rad}(I)$, and so $M=\operatorname{rad}(u, v)$. 
Proposition 3.3. Let $R$ be a Hilbert domain of finite character, $R[X]$ the polynomial ring over $R$, and let $M^{*}$ be any maximal ideal of $R[X]$. Then the least number of generators among the ideals in $R[X]$ with radical $M^{*}$ is two.

Proof. Let $M^{*}$ be any maximal ideal of $R[X]$. Then, by $[\mathbf{1 5}$, Theorem 5], $M=M^{*} \cap R$ is a maximal ideal in $R$. Hence, $M^{*}=(M, f)$ for some monic polynomial $f$ in $M^{*}$. Now, by adapting the proof of the last part of Theorem 2.3, we obtain $M^{*}=\operatorname{rad}(u, g)$ for some element $u$ in $M$ and some polynomial $g$ in $M^{*}$. Next we show that $M^{*}$ cannot be the radical of a single element. For, if $M^{*}=\operatorname{rad}(h)$ for some $h$ in $M^{*}$, then it is clear that $h$ must be a constant $c$. Since the monic polynomial $f$ is in $M^{*}, f^{k}$ is in (c) for some positive integer $k$. But then $c$ divides all the coefficients of $f^{k}$, and hence it divides the highest coefficient 1 . This is a contradiction to the fact that $M^{*}$ is a maximal ideal. Therefore, the least number of generators among the ideals in $R[X]$ with radical $M^{*}$ is two.

Corollary 3.4. Let $R$ be a Hilbert domain of finite character and $R[X]$ the polynomial ring over $R$. Then, each maximal ideal of $R[X]$ of maximal height is radically perfect if and only if $R[X]$ is of dimension two.

Proof. This follows from Proposition 3.3. It is worth noting that, without radically perfectness assumption, one can only conclude from Proposition 3.2 that $\operatorname{dim}(R[X]) \geq 2$.

Proposition 3.5. Let $R$ be a Noetherian Hilbert domain of finite character which contains a field of characteristic zero. Then, each prime ideal of $R[X]$ is radically perfect if and only if $R$ is a Dedekind domain with torsion ideal class group.

Proof. By Proposition 3.2, $R$ is of dimension one, and by $[\mathbf{1 0}$, Theorem 2.2], $R$ is integrally closed but then the statement follows from $[\mathbf{9}$, Theorem 2.1].

Proposition 3.6. For a ring $R$, the following statements are equivalent: 
(a) $R$ is a Noetherian Hilbert ring of finite character with zero divisors.

(b) $R$ is Artinian.

(c) $R$ is Noetherian and each ideal of $R[X]$ is the radical of a principal ideal.

Proof. $(\mathrm{a}) \Rightarrow(\mathrm{b})$. By the proof of Proposition 3.2, we have each prime ideal of $R$ is maximal, and thus $R$ is a Noetherian ring of dimension zero, and so is Artinian.

(b) $\Rightarrow(\mathrm{a})$. This is obvious.

(b) $\Rightarrow(\mathrm{c})$. Follows from $[\mathbf{9}$, Remark 2.3].

$(\mathrm{c}) \Rightarrow(\mathrm{b})$. The condition implies that $R$ is a Noetherian ring of dimension zero, and so is Artinian.

Theorem 3.7. Let $R$ be a Hilbert domain in which each nonzero ideal is contained in finitely many maximal ideals. Then, each invertible maximal ideal of $R$ being radically perfect implies that a positive power of each invertible radical ideal is principal.

Proof. By Proposition 3.2, we have $R$ is of dimension one. Let $M$ be an invertible maximal ideal of $R$. Then $M_{M}$ is principal in $R_{M}$ and $M_{M}$ being the only nonzero prime ideal of $R_{M}$ implies that $R_{M}$ is Noetherian, and so is a DVR. Since $M$ is radically perfect, $M=\operatorname{rad}(c)$ for some $c \in M$, and so (c) is $M$-primary. Note that as (c) is $M$-primary, (c) $=c R_{M} \cap R$ and as $M^{r}$ is primary for all natural numbers $r$ we have $M^{r} R_{M} \cap R=M^{r}$. Now, as $R_{M}$ is a rank one DVR, $M^{n} R_{M}=c R_{M}$ for some natural number $n$, and from the above observations $M^{n}=(\mathrm{c})$. Now let $I$ be an invertible radical ideal of $R$. Then $I=\operatorname{rad}(I)=M_{1} \cdots M_{n}$. Since $I$ is invertible, it follows that each $M_{i}$ is invertible. But then a power of each $M_{i}$ is principal and, from this, we see that a positive power of $I$ is principal.

Let $R$ be a ring, and let $\operatorname{CopRad}(R)$ denote the multiplicative semigroup generated by the set of pairwise coprime radical ideals of $R$ that are invertible. Then each generator of CopRad $(R)$ represents a class of finitely generated projective $R$-modules of rank one. Note that 
for any two generators $I, J$ of $\operatorname{CopRad}(R), \operatorname{rad}(I J)=\operatorname{rad}(I \cap J)=$ $\operatorname{rad}(I) \cap \operatorname{rad}(J)=I \cap J=I J$, this semigroup has an identity, and the identity is the ring $R$ itself. Clearly, $\operatorname{CopRad}(R)$ is a multiplicative subsemigroup of the Picard group of $R$.

Theorem 3.8. Let $R$ be a Hilbert domain in which each nonzero ideal is contained in finitely many maximal ideals. Then each invertible maximal ideal of $R$ is radically perfect if and only if $\operatorname{CopRad}(R)$ is torsion.

Proof. The only if part follows from Theorem 3.7. For the if part, take $M$ to be any invertible maximal ideal of $R$. Then $M$ is in $\operatorname{CopRad}(R)$, and hence $M^{k}=(c)$ for some $c$ in $R$ and some positive integer $k$. But then it is clear that $M=\operatorname{rad}(c)$ and, by Proposition 3.2, $R$ is of dimension one. Therefore, $M$ is radically perfect.

We remark that Theorems 3.7 and 3.8 are true for any integral domain of dimension one. Since in their proofs, the condition "Hilbert" is used to ensure that the dimension of the ring is one.

\section{Radically perfect prime ideals in Krull domains of finite} character. Here, we investigate radically perfectness of prime ideals in Krull domains, and one of our main results of this section is the following theorem.

Theorem 4.1. Let $R$ be a two-dimensional Krull domain of finite character. Then each prime ideal $P$ of $R$ is radically perfect if and only if the divisor class group of $R$ is torsion.

Proof. The only if part follows from the fact that, in a Krull domain $R$, each height one prime ideal is the radical of a principal ideal if and only if the divisor class group of $R$ is torsion [1, Theorem 3.2].

The proof of the if part is as follows. Since we are assuming that the divisor class group of $R$ is torsion, each height one prime ideal of $R$ is the radical of a principal ideal and hence is radically perfect. So, we are left to consider the case of the prime ideals of height two. Let $M$ 
be a height two prime ideal of $R$. Then, by Proposition 2.2, there are elements $u, v$ in $M$ such that $M=j-\operatorname{rad}(u, v)$. Clearly, $\operatorname{rad}(u, v)$ is contained in $M$, and so any prime ideal of $R$ that contains $u$ and $v$ is contained in $M$. Therefore, if no prime ideal of $R$ contained in $M$ contains $u$ and $v$, then $M=\operatorname{rad}(u, v)$. So, suppose that there is a prime ideal $N$ of $R$ containing $(u, v)$. Then, necessarily, $N$ is properly contained in $M$ and so is of height one. Therefore, $N=\operatorname{rad}(\mathrm{c})$ for some $c \in N$. Since $N$ contains $u$ and $v$ and $N=\operatorname{rad}(\mathrm{c})$, some powers of $u, v$ are in (c). Let $x$ be an element in $M$ which is not in $N$. Then clearly the ideal $(c, x)$ is contained only in $M$ and not contained in any other prime ideal of $R$. For, if $P$ is any other prime ideal of $R$ containing $c$ and $x$, then $N$ must be strictly contained in $P$, as $x$ is not in $N$, which implies that $P$ is of height two and it contains $u$ and $v$. Therefore, $P$ must be nothing other than $M$. Hence, $M=\operatorname{rad}(c, x)$. In order to conclude that $M$ is radically perfect, we need to show that $M$ is not the radical of a principal ideal. Suppose that $M=\operatorname{rad}(b)$ for some $b \in M$. Then, since $R$ is a Krull domain, it follows from the corollary to Theorem 12.3 of [19] that $R b=P_{1}^{\left(n_{1}\right)} \cap P_{2}^{\left(n_{2}\right)} \cap \cdots \cap P_{k}^{\left(n_{k}\right)}$ for some finite number of height one prime ideals of $R$ where $P_{i}^{\left(n_{i}\right)}$ are the symbolic powers of $P_{i}(1 \leq i \leq n)$. But then, clearly, $M=\operatorname{rad}(b)=\operatorname{rad}\left(P_{1}^{\left(n_{1}\right)} \cap P_{2}^{\left(n_{2}\right)} \cap \cdots \cap P_{k}^{\left(n_{k}\right)}\right)=$ $\operatorname{rad}\left(P_{1}^{\left(n_{1}\right)}\right) \cap \operatorname{rad}\left(P_{2}^{\left(n_{2}\right)}\right) \cap \cdots \cap \operatorname{rad}\left(P_{k}^{\left(n_{k}\right)}\right)=P_{1} \cap P_{2} \cap \cdots \cap P_{n} \subseteq P_{i}$ for each $(1 \leq i \leq n)$. This is a contradiction to the fact that $M$ is of height two. Therefore, the least number among the generators of ideals with radical $M$ in $R$ is two, and so $M$ is radically perfect.

Corollary 4.2. Let $R$ be a two-dimensional unique factorization domain of finite character. Then each prime ideal of $R$ is radically perfect.

Proof. The proof follows from the above proof by noting that every unique factorization domain is a Krull domain and that in a unique factorization domain height one primes are principal.

Corollary 4.3. Let $R$ be a two-dimensional locally factorial Krull domain of finite character. Then the following statements are equivalent: 
(a) Each height one prime ideal of $R[X]$ is radically perfect.

(b) The divisor class group of $R$ and of $R[X]$ are torsion.

(c) A positive power of each height one prime ideal of $R$ is principal.

(d) A positive power of each divisorial ideal of $R$ is principal.

(e) Each prime ideal of $R$ is radically perfect.

Proof. (a) $\Leftrightarrow(\mathrm{b})$. Each height one prime ideal of $R[X]$ is radically perfect if and only if the divisor class group of $R[X]$ is torsion which is so if and only if the class group of $R$ is torsion.

(b) $\Rightarrow$ (c). Let $P$ be a height one prime ideal of $R$. Since $R$ is a locally factorial Krull domain, by [1, Theorem 3.1], $P$ is invertible and, since the divisor class group of $R$ is torsion, by [1, Theorem 3.2], $P^{(n)}$ is principal for some $n>0$. Since, for such a prime $P$, we have $P^{(n)}=P^{n}$, and also $P^{n}$ is principal.

(c) $\Rightarrow$ (d). Let $I$ be a divisorial ideal in $R$. Then $R$ is Krull, $I=P_{1}^{\left(n_{1}\right)} \cap \cdots \cap P_{k}^{\left(n_{k}\right)}$ where $P_{1}, \ldots, P_{k}$ are height one primes and since, by assumption, a power of each $P_{i}$ is principal, it follows that a power of $I=P_{1}^{\left(n_{1}\right)} \cap \cdots \cap P_{k}^{\left(n_{k}\right)}=P_{1}^{n_{1}} \cdots P_{k}^{n_{k}}$ is principal.

$(\mathrm{d}) \Rightarrow(\mathrm{e})$. Since in a Krull domain each height one prime ideal is divisorial, it follows that a power of each height one prime ideal is principal and also is radically perfect. The case of height two primes being radically perfect follows from Theorem 4.1.

$(e) \Rightarrow(b)$. This is nothing but Theorem 4.1.

Corollary 4.4. Let $R$ be a two-dimensional locally factorial Krull domain of finite character. Then each prime ideal of $R[X]$ is radically perfect implies that the ring $R(X)$, the localization of $R[X]$ at the set of polynomials with unit content, is a unique factorization domain.

Proof. This follows from the above Corollary 4.3 and [1, Theorem $3.1]$.

Proposition 4.5. Let $R$ be a two-dimensional Krull domain of finite character. Suppose that the divisor class group of $R$ is torsion. Then each maximal ideal of $R[X]$ of maximal height is the radical of an ideal generated by at most three elements. 
Proof. Let $M^{*}$ be a maximal ideal of $R[X]$ of maximal height such that $M^{*} \cap R=M$. Then it follows from the proof of Theorem 2.3 that $M$ is a maximal ideal of $R$. Hence, $M^{*}=(M, f)$ for some monic polynomial $f$ in $M^{*}$ and so it follows from the proof of Theorem 4.1 that $M^{*}$ is the radical of an ideal generated by at most three elements.

We now make the following observation:

Let $R$ be a two-dimensional Noetherian domain of finite character that contains a field of characteristic zero. Suppose that each prime ideal of $R[X]$ is radically perfect. Then it follows from $[\mathbf{1 0}$, Theorem 2.2 ] that $R$ is normal. Since Noetherian normal domains are Krull, $R$ is a Krull domain and therefore $R[X]$ is a Krull domain having the property that each prime ideal of it is radically perfect. Hence, each height one prime ideal of $R[X]$ is the radical of a principal ideal and therefore by [1, Theorem 3.2], the divisor class group of $R[X]$ is torsion. Knowing that the divisor class group of $R$ and that of $R[X]$ are isomorphic, we see that the divisor class group of $R$ is torsion, and hence, by Theorem 4.1, each prime ideal of $R$ is radically perfect.

Conversely, suppose now that $R$ is a two-dimensional Noetherian Krull domain of finite character whose divisor class group is torsion. Then, for any nonzero prime ideal $P^{*}$ of $R[X]$, we have the following three cases.

Case 1. ht $\left(P^{*}\right)=3$. Then Proposition 4.5 and the fact that $R$ is Noetherian imply $P^{*}$ is radically perfect.

Case 2. ht $\left(P^{*}\right)=1$. Since the divisor class group of $R$, and hence that of $R[X]$, are torsion, it follows from [1, Theorem 3.2] that $P^{*}$ is radically perfect.

Case 3. ht $\left(P^{*}\right)=2$. Then either ht $\left(P^{*} \cap R\right)=2$, in which case $P^{*}$ is radically perfect, or ht $\left(P^{*} \cap R\right)=1$, in which case $R / P^{*} \cap R$ is a Noetherian semilocal domain of dimension one. Hence, by $[\mathbf{1 2}$, Proposition 3], each prime ideal of $\left(R / P^{*} \cap R\right)[X]$, except perhaps those primes contracting to zero in $R / P^{*} \cap R$, are radically perfect and each maximal ideal of $\left(R / P^{*} \cap R\right)[X]$ is the $j$-radical of a single element.

The following result is related to the above Proposition 4.5 which correlates radically perfectness of the prime ideals of $R$ to radically 
perfectness of maximal ideals of Int $(R)$, the ring of integer-valued polynomials over $R$.

We recall that $\operatorname{Int}(R):=\{f \in K[X]: f(R) \subseteq R\}$ and, for any $\alpha$ in $R$ and any prime ideal $P$ of $R$, the set $\mathcal{B}(\alpha, P)=\{f \in \operatorname{Int}(R): f(\alpha) \in P\}$ is a prime ideal of $\operatorname{Int}(R)$ and, if $P$ is a maximal ideal of $R$, then $\mathcal{B}(\alpha, P)$ is a maximal ideal of $\operatorname{Int}(R)[\mathbf{3}]$. Note that, in the following statement, $R$ is not assumed to be of finite character.

Proposition 4.6. Let $R$ be a two-dimensional Noetherian Krull domain whose divisor class group is torsion. Then, each maximal ideal of $\operatorname{Int}(R)$ is radically perfect implies that each prime ideal $P$ of $R$ is either radically perfect or is the radical of ht $(P)+1$ elements.

Proof. Let $P$ be a prime ideal of $R$. Then, either $P$ is of height one or it is of height two. If the height of $P$ is one, then since the divisor class group of $R$ is torsion, $P=\operatorname{rad}(a)$ for some $a \in P$. Therefore, $P$ is radically perfect. If, on the other hand, the height of $P$ is two, then there exists a chain $(0) \subsetneq P_{1} \subsetneq P$ of prime ideals of $R$ of length three which gives rise to a chain $(0) \subsetneq \mathcal{B}(\alpha,(0)) \subsetneq \mathcal{B}\left(\alpha, P_{1}\right) \subsetneq \mathcal{B}(\alpha, P)$ of prime ideals in $\operatorname{Int}(R)$. From this, it follows that $h t(\mathcal{B}(\alpha, P)) \geq 3$. Now, since Krull domains are locally essential domains, it follows from [13, Theorem 2.1] that $\operatorname{dim}(\operatorname{Int} R)=\operatorname{dim} R[X]$. But then $R$ being Noetherian gives that $\operatorname{dim}(\operatorname{Int}(R))=\operatorname{dim} R[X]=3$, and so $\mathcal{B}(\alpha, P)$ is a maximal ideal of maximal height in $\operatorname{Int}(R)$. Hence, by the hypothesis, we have $\mathcal{B}(\alpha, P)=\operatorname{rad}\left(f_{1}, f_{2}, f_{3}\right)$ for some $f_{1}, f_{2}, f_{3} \in \mathcal{B}(\alpha, P)$ from which it follows that $\left(f_{1}(\alpha), f_{2}(\alpha), f_{3}(\alpha)\right) \subseteq P$. Let $Q$ be any other prime ideal of $R$ containing $\left(f_{1}(\alpha), f_{2}(\alpha), f_{3}(\alpha)\right)$. Then, in Int $(R)$, $\mathcal{B}(\alpha, Q)$ contains the ideal $\left(f_{1}, f_{2}, f_{3}\right)$ and so it contains $\mathcal{B}(\alpha, P)$. Since $\mathcal{B}(\alpha, P)$ is maximal, we have $\mathcal{B}(\alpha, Q)=\mathcal{B}(\alpha, P)$, and so $P=Q$ (here we use the fact that $P \subseteq \mathcal{B}(\alpha, P)$ for any prime ideal $P$ of $R$ and that if $Q$ is a prime ideal of $R$ with $Q \subseteq \mathcal{B}(\alpha, P)$, then $Q \subseteq P)$. Therefore, it follows that $P=\operatorname{rad}\left(f_{1}(\alpha), f_{2}(\alpha), f_{3}(\alpha)\right)$, and hence $P$ is the radical of an ideal generated by ht $(P)+1$ elements.

\section{Radically perfect prime ideals in Prüfer domains of finite} character. Here we show that each prime ideal of a Prüfer domain $R$ or of the polynomial ring $R[X]$ over $R$ being radically perfect implies 
that $R$ is of dimension at most two and in some special cases is of dimension one.

Proposition 5.1. Let $R$ be a finite dimensional Prüfer domain of finite character. Then each maximal ideal of $R$ is radically perfect implies that $R$ is of dimension at most two.

Proof. Since $R$ is of finite character, for each maximal ideal $M$ of $R$, $M=j-\operatorname{rad}(u, v)$. Now $R$ being Prüfer implies that $\operatorname{rad}(u, v)$ is a prime ideal of $R$ with $M=j-\operatorname{rad}(\operatorname{rad}(u, v))$. Hence, $R / \operatorname{rad}(u, v)$ is a valuation ring with maximal ideal $M / \operatorname{rad}(u, v)$. If $R / \operatorname{rad}(u, v)$ is a field, then $M=\operatorname{rad}(u, v)$. If, however, $R / \operatorname{rad}(u, v)$ is not a field, then it follows that $M / \operatorname{rad}(u, v)$ is nonzero, in which case $M=\operatorname{rad}(x)$ for some $x \in M-\operatorname{rad}(u, v)$. From this, it follows that $R$ is of dimension at most two.

The next result is on a special type of Prüfer domain whose definition is as follows: An integral domain $R$ is a QR-domain if each overring of it is a quotient ring. Recall that a Prüfer domain is a QR-domain if and only if the radical of a finitely generated ideal is the radical of a principal ideal [20].

Proposition 5.2. Let $R$ be a finite dimensional $Q R$-domain of finite character. Then each maximal ideal of $R$ is the radical of a principal ideal.

Proof. Let $M$ be any maximal ideal of $R$. Since $R$ is a QRdomain of finite character, it follows from the above proof that $M=$ $j-\operatorname{rad}(\operatorname{rad}(u, v))$ for some $u, v \in M$ and, as $\operatorname{rad}(u, v)=\operatorname{rad}(c)$ for some $c \in M, R / \operatorname{rad}(c)$ is a valuation ring and, from this, it follows that $M$ is the radical of a principal ideal.

In order to appreciate the content of the next statement, we remark that, if $R=K[Y, Z]$ where $K$ is a field of characteristic zero and $P^{*}$ is a prime ideal of $R[X]$ of height two with $P^{*} \cap R=P$ a height one prime ideal of $R$, then $R / P$ is an integral domain of dimension one. If $R / P$ is not integrally closed, then not every prime ideal of $(R / P)[X]$ 
is radically perfect. As an example, take $P^{*}=\left(Y^{2}-Z^{3}, X\right)$. Then $P^{*} \cap R=P=\left(Y^{2}-Z^{3}\right)$ is a prime ideal of height one in $R$ and $R / P$ is not integrally closed. Therefore, not every prime ideal of $(R / P)[X]$ is radically perfect (see $[\mathbf{1 0}$, Theorem 2.2]).

Proposition 5.3. Let $R$ be a Prüfer domain of finite character. Suppose that each prime ideal of $R[X]$ is radically perfect. Then either $R$ is of dimension one and each prime ideal of $R$ is the radical of a principal ideal or $R$ is of dimension two and for each height one prime ideal $P$ of $R$, each prime ideal of $(R / P)[X]$ is radically perfect.

Proof. Let $M$ be a maximal ideal of maximal height in $R$. Let $P$ be a prime ideal of height one which is contained in $M$. Then, as $P R[X]$ is radically perfect, $P=\operatorname{rad}(c)$ for some $c \in P$. Since $R$ is of finite character, $c$ is contained in only finitely many maximal ideals and, therefore, $R / P$ is a semilocal Prüfer domain and hence is Bezout. By [8, Corollary 2], each maximal ideal of $R / P$ is the radical of a principal ideal. Hence $M / P=\operatorname{rad}(\bar{d})$ for some $\bar{d} \in M / P$, but then $M=\operatorname{rad}(c, d)$ where $d$ is the preimage of $\bar{d}$ in $R$, from which we obtain $M R[X]=\operatorname{rad}(c R[X]+d R[X])$. Therefore, ht $(M)=\operatorname{ht}(M R[X]) \leq 2$, and hence $\operatorname{dim}(R) \leq 2$. But then $R / P$ is of dimension at most one. If $R / P$ is a field, then $R$ is of dimension one and each prime ideal of $R$ is the radical of a principal ideal. If, on the other hand, $R / P$ is of dimension one, then $R$ is of dimension two and by [9, Theorem 2.5], each prime ideal of $(R / P)[X]$ is radically perfect.

We failed in our attempt to see whether or not height one prime ideals in the polynomial ring $R[X]$ over a Prüfer domain $R$ contracting to zero in $R$ are radically perfect. Such primes are known to be invertible. This is a consequence of the fact that every Prüfer domain is a generalized GCD domain and the primes of $R[X]$ contracting to zero in a generalized GCD domain $R$ are invertible (see [2, Theorem $15])$.

6. The relation between coprimely packed and radically perfect ideals. We begin this section by recalling that the set of prime ideals of a ring $R$ is coprimely packed if, whenever a prime ideal 
$P$ of $R$ is contained in the union of a family of maximal ideals of $R$, then $P$ is contained in one of the maximal ideal in the family [5]. We also note that, if $R$ is an integral domain of dimension one in which each nonzero ideal is contained in finitely many maximal ideals, then the condition that Spec $R$ is coprimely packed implies that CopRad $(R)$ is torsion. The justification of this fact is the same as the one given in the proof of Theorem 3.8.

Before stating our next result, we recall that an integral domain is an S-domain if, for every prime ideal $P$ of $R$ of height one, the prime ideal $P R[X]$ of $R[X]$ is also of height one.

Proposition 6.1. Let $R$ be an S-domain of dimension one. Then each prime ideal of $R[X]$ that does not contract to zero ideal of $R$ is radically perfect if and only if MaxSpec $R$ is coprimely packed.

Proof. Let $M$ be a maximal ideal of $R$. Then the prime ideal $M R[X]$ of $R[X]$ is radically perfect and so $M R[X]$ is the radical of a constant $c$ in $R[X]$, which clearly implies that $M=\operatorname{rad}(c)$ in $R$. Therefore, MaxSpec $R$ is coprimely packed. For the converse, let $P^{*}$ be a prime ideal of $R[X]$ such that $P^{*} \cap R=P$ is a nonzero prime ideal in $R$. Since MaxSpec $R$ is coprimely packed, $P=\operatorname{rad}(a)$ for some $a$ in $P$. Now we either have $P^{*}=P R[X]$ or $P R[X]$ is strictly contained in $P^{*}$. If $P^{*}=P R[X]$, then $P^{*}=\operatorname{rad}(a)$ in $R[X]$. If on the other hand, $P^{*}$ strictly contains $P R[X]$, then $P^{*}$ is a maximal ideal of height two, and therefore $P^{*}=\operatorname{rad}(a, f)$ in $R[X]$ for some monic polynomial $f$. Now use the argument of the proof of Proposition 3.3 to see that $P^{*}$ cannot be the radical of a principal ideal, and so is radically perfect.

Proposition 6.2. Let $R$ be a finite-dimensional integral domain. If MaxSpec $R$ is coprimely packed, then each maximal ideal $M^{*}$ of $R[X]$ of maximal height is the $j$-radical of an ideal generated by two elements. Moreover, if in addition $R$ is Hilbert, then the condition implies that each maximal ideal of $R[X]$ is the radical of an ideal generated by two elements.

Proof. Let $M^{*}$ be a maximal ideal of $R[X]$ of maximal height. Then use the proof of Theorem 2.3 to see that $M^{*} \cap R=M$ is a maximal 
ideal of $R$. Hence, $M^{*}=(M, f)$ for some monic polynomial $f \in M^{*}$. Since MaxSpec $R$ is coprimely packed, $M=j-\operatorname{rad}(m)$ for some $m \in M$, and hence $j-\operatorname{rad}(m, f)$ is contained in $M^{*}$. If now $Q^{*}$ is any maximal ideal of $R[X]$ containing the ideal $(m, f)$, then, as in the proof of Theorem 2.3, we see that $Q^{*} \cap R=M$; hence, $Q^{*}=M^{*}$. Therefore, $M^{*}=j-\operatorname{rad}(m, f)$. In the case of $R$ being Hilbert, all we need to note is that if $M^{*}$ is any maximal ideal (not just a maximal ideal of maximal height) of $R[X], M^{*} \cap R$ is a maximal ideal of $R$ and that $j-\operatorname{rad}(I)=\operatorname{rad}(I)$ for any ideal $I$ in $R$.

Corollary 6.3. Let $R$ be a finite-dimensional Hilbert domain with coprimely packed set of maximal ideals. Then each maximal ideal of $R[X]$ is radically perfect if and only if the height of each maximal ideal of $R[X]$ is two.

Proof. Let $M^{*}$ be a maximal ideal of $R[X]$. Then, by Proposition 6.2, it follows that $M^{*}=\operatorname{rad}(m, f)$ where $m$ is a constant and $f$ is a monic polynomial. Next, we use the proof of Proposition 3.3 and see that $M^{*}$ cannot be the radical of a principal ideal. Since $M^{*}$ is radically perfect and is the radical of an ideal generated by two elements, it is of height two.

The converse also follows from the proof of Proposition 6.2.

Proposition 6.4. If $R$ is a finite-dimensional local integral domain, then each maximal ideal of maximal height of $R[X]$ is the $j$-radical of a principal ideal.

Proof. Let $M^{*}$ be any maximal ideal of $R[X]$ of maximal height. Then $M^{*} \cap R=M$ is the maximal ideal of $R$, and so $M^{*}=(M, f)$ for some monic polynomial $f \in M^{*}$. Clearly, any maximal ideal $N^{*}$ of $R[X]$ containing $f$ has to contain $M$, and therefore $M^{*}=j-\operatorname{rad}(f)$.

Proposition 6.5. Let $R$ be a finite-dimensional valuation ring with maximal ideal $M$. If $M R[X]$ is radically perfect, then $\operatorname{Spec} R[X]$ is coprimely packed. 
Proof. Let $R$ be a finite-dimensional valuation ring with maximal ideal $M$. Then $M=\operatorname{rad}(c)$ for some $c \in M$, which implies that $M R[X]=\operatorname{rad}(c)$ in $R[X]$. Since $M R[X]$ is radically perfect, ht $(M R[X])=\operatorname{ht}(M)=1$. Therefore, $R$ is of dimension one.

Let $P^{*}$ be any prime ideal of $R[X]$. Then either $P^{*} \cap R$ is zero, or is the maximal ideal $M$ of $R$. If $P^{*} \cap R=0$, then, as in the proof of Theorem 4.1 of $[\mathbf{1 0}], P^{*}=\operatorname{rad}(f)$ for some polynomial $f$ in $R[X]$. But then it is clear that $P^{*}$ is coprimely packed. If, however, $P^{*} \cap R=M$, then we either have $P^{*}=M R[X]$, in which case $P^{*}=\operatorname{rad}(c)$ in $R[X]$ or $P^{*}=(M, f)$ for some monic polynomial $f$. The case $P^{*}=\operatorname{rad}(c)$ clearly implies that $P^{*}$ is coprimely packed. If, on the other hand, $P^{*}=(M, f)$, then by Proposition $6.4, P^{*}=j-\operatorname{rad}(f)$ and so is coprimely packed.

Theorem 6.6. For a finite dimensional $Q R$-domain $R$, the following statements are equivalent:

(a) For each maximal ideal $M$ of $R$, the prime ideal $M R[X]$ of $R[X]$ is radically perfect.

(b) $R$ is of dimension one and $\operatorname{MaxSpec} R$ is coprimely packed.

(c) Each prime ideal of $R[X]$ is radically perfect.

Proof. (a) $\Rightarrow$ (b). Let $M$ be any maximal ideal of $R$ and ht $(M R[X])=\operatorname{ht}(M)=n$. Then $M R[X]=\operatorname{rad}\left(f_{1}, \ldots, f_{n}\right)$ for some $f_{1}, \ldots, f_{n} \in M R[X]$. But then it is easy to see that $M R[X]=$ $\operatorname{rad}\left(A_{f_{i}}, \ldots, A_{f_{n}}\right)$ in $R[X]$ where $A_{f_{i}}$ is the content ideal of $f_{i}$. Since $\operatorname{rad}\left(A_{f_{i}}, \ldots, A_{f_{n}}\right)=\operatorname{rad}(a)$ in $R$ for some $a \in R$, it follows that $M R[X]=\operatorname{rad}(a)$ in $R[X]$ and that $M=\operatorname{rad}(a)$ in $R$. Thus, ht $(M R[X])=\operatorname{ht}(M)=1$. Hence, $R$ is of dimension one and MaxSpec $R$ is coprimely packed.

(b) $\Rightarrow$ (c). Let $P^{*}$ be any prime ideal of $R[X]$ and $P^{*} \cap R=P$. Then we have three cases to consider:

Case 1. ht $\left(P^{*}\right)=2$ in which case, $P \neq 0$ and so $P^{*}=(P, f)=$ $\operatorname{rad}(c, f)$ where $P=\operatorname{rad}(c)$ and $f$ is a monic polynomial in $P^{*}$. As in the proof of Proposition 3.3, $P^{*}$ cannot be written as the radical of a principal ideal and therefore is radically perfect.

Case 2. ht $\left(P^{*}\right)=1$ and $P \neq 0$, in which case $P^{*}=P R[X]=\operatorname{rad}(c)$ in $R[X]$ where $P=\operatorname{rad}(c)$ in $R$, and so $P^{*}$ is radically perfect. 
Case 3. ht $\left(P^{*}\right)=1$ and $P=0$ in this case by Theorem 15 of $[\mathbf{2}], P^{*}$ is invertible, and therefore is finitely generated. Let $P^{*}=\left(f_{1}, \ldots, f_{n}\right)$ and $A_{f_{i}}$ be the content ideal of $f_{i}$. Then using the proof of $(\mathrm{a}) \Rightarrow$ (b) above, we see that $P^{*}=\operatorname{rad}(a)$ for some $a \in R$ and therefore is radically perfect.

(c) $\Rightarrow$ (a). This is clear.

Theorem 6.7. Let $R$ be a Prüfer domain in which each maximal ideal is finitely generated and whose set of maximal ideals is coprimely packed. Then, for each maximal ideal $M$ of $R$, the prime ideal $M R[X]$ of $R[X]$ is radically perfect if and only if $R$ is a Dedekind domain with torsion ideal class group.

Proof. By [11, Theorem 3.1], $R$ is of dimension one and so $R$ is a Dedekind domain. But then the result follows from $[\mathbf{9}$, Theorem 2.1].

We conclude this paper by noting that, in general, the property that MaxSpec $R$ is coprimely packed does not imply that each prime ideal of $R$ is radically perfect. As an example, take $R$ to be a valuation domain of dimension greater than one. It is also not the case that each prime ideal of $R$ being radically perfect implies that MaxSpec $R$ is coprimely packed. As an example, take $R$ to be the polynomial ring $\mathbf{Z}[X]$ over the ring of integers $\mathbf{Z}$ ([7, Theorem 1.3] and [9, Theorem 2.1]). However, if $R$ is an integral domain of dimension one, then MaxSpec $R$ is coprimely packed if and only if each prime ideal of $R$ is radically perfect. Moreover, either of these conditions imply that $G(R)$, the semigroup generated by the set of invertible pairwise coprime radical ideals that are contained in finitely many maximal ideals is torsion (see the argument at the beginning of this section). Semilocal integral domains of dimension one are examples of such domains. Note that, as used in the text, $G(R)$ is in general a multiplicative subsemigroup of $\operatorname{CopRad}(R)$.

Acknowledgments. We wish to thank the referee for his/her helpful suggestions.

\section{REFERENCES}

1. D.D. Anderson, Globalization of some local properties in Krull domains, Proc. Amer. Math. Soc. 85 (1982), 141-145. 
2. D.D. Anderson, T. Dumitrescu and M. Zafrullah, Quasi-Schreier domains II, Comm. Alg. 35 (2007), 2096-2104.

3. P.-J.Cahen, Integer valued polynomials on a subset, Proc. Amer. Math. Soc. 117 (1993), 919-929.

4. D. Eisenbud and E.G. Evans, Every algebraic set in $n$-space is the intersection of n hypersurfaces, Inv. Math. 19 (1973), 107-112.

5. V. Erdoğdu, Coprimely packed rings, J. Number Theor. 28 (1988), 1-5.

6. - The prime avoidance of ideals in Noetherian Hilbert rings, Comm. Alg. 22 (1994), 4989-4990.

7. - The prime avoidance of maximal ideals in commutative rings, Comm. Alg. 23 (1995), 863-868.

8. — Three notes on coprime packedness, J. Pure Appl. Alg. 148 (2000), $165-170$.

9. - Coprime packedness and set theoretic complete intersections of ideals in polynomial rings, Proc. Amer. Math. Soc. 132 (2004), 3467-3471.

10. - Radically perfect prime ideals in polynomial rings, Arch. Math. 93 (2009), 213-217.

11. - Efficient generation of prime ideals in polynomial rings up to radical, Comm. Alg. 38 (2010), 1802-1807.

12. V. Erdoğdu and S. McAdam, Coprimely packed Noetherian polynomial rings, Comm. Alg. 22 (1994), 6459-6470.

13. M. Fontana and S. Kabbaj, Essential domains and two conjectures in dimension theory, Proc. Amer. Math. Soc. 132 (2004), 2529-2535.

14. R. Gilmer, Multiplicative ideal theory, Queen's Papers Pure Appl. Math. 90, Queen's University, Kingston Ontario, 1992.

15. O. Goldman, Hilbert rings and the Hilbert Nullstellensatz, Math. Z. 54 (1951), $136-140$.

16. I. Kaplansky, Commutative rings, University of Chicago Press, Boston, 1974.

17. E. Kunz, Introduction to commutative algebra and algebraic geometry, Birkhauser, Boston, 1985.

18. G. Lyubeznik, The number of defining equations of affine algebraic sets, Amer. J. Math. 114 (1992), 413-463.

19. H. Matsumura, Commutative ring theory, Cambridge University Press, Cambridge, 1986.

20. R. Pendleton, A characterization of Q-domains, Bull. Amer. Math. Soc. 72 (1966), 499-500.

Department of Mathematics, Istanbul Technical University, Maslak 80626, Istanbul, Turkey

Email address: erdogdu@itu.edu.tr

Department of Mathematics, Istanbul Technical University, Maslak 80626, Istanbul, Turkey

Email address: harman@itu.edu.tr 